Proc. Indian Acad. Sci, Vol, 88 A, Part I, Number 6. December 1979, pp. 445 452,

(C) printed in India.

\title{
A study of interaction in the lowest singlet and triplet states of $\mathbf{H}_{2}$
}

\author{
K L SEBASTLAN and A K CHANDRA \\ Department of Inorganic and Physical Chemistry, Indian Institute of Science, \\ Bangalore 560012 \\ MS received 11 July 1979
}

\begin{abstract}
The potential energy curves of the ground state and the first excited state of $\mathrm{H}_{2}$ are examined in terms of the electronic force acting on each nucleus. The results reveal the detailed course of events that occur when two hydrogen atoms with parallel and antiparallel electron spins approach one another from a large internuclear separation.
\end{abstract}

Keywords. Potential energy curves; ground state; excited state: atomic polarisation ; orbital contraction.

\section{Introduction}

In our previous studies (Bader and Chandra 1968; Chandra and Sunder 1971; Sunder and Chandra 1974; Chandra and Sebastian 1976a,b) we have presented an interpretation of the prccess of bond-formation from two separated atoms as a function of internuclear separation in terms of the electronic force acting on the nuclei and the change in molecular charge distributions. Such studies reveal the nature and origin of both the long-range and short-range interactions. Although we have presented earlier the detailed course of events that occur during the formation of the ground state of $\mathrm{H}_{2}$ (Bader and Chandra 1968) from two ground-state hydrogen atoms, it is imperative to study how two ground-state hydrogen atoms with their electron spins parallel behave when they approach each other from an infinite separation. Since the triplet state of $\mathrm{H}_{2}$ i.e. ${ }^{3} \Sigma_{u}^{+}$is unstable at all separations such study along with the studies of the ${ }^{1} \Sigma_{g}^{+}$state of $\mathrm{H}_{2}$ may provide interesting clues as to the nature of binding and are also relevant to theories of molecular structure and intermolecular forces. The object of the present investigation is to examine in terms of the eleatronic forces acting on the nuclei the potential energy curves for both the ground state $\left({ }^{1} \Sigma_{g}^{+}\right)$and the first excited state $\left({ }^{3} \Sigma_{w}^{+}\right)$of $\mathrm{H}_{2}$ which were obtained with high precision by Bowman et al (1970). They employed the extended Hartree-Fock approximation for the ${ }^{1} \Sigma_{0}^{+}$state and the usual singleconfiguration Hartree-Fock approximation for the ${ }^{3} \Sigma_{u}^{+}$state. Both states of $\mathrm{H}_{2}$ dissociate into two grcund-state hydrogen atoms and their wave functions involve $\sigma_{\theta}$ and $\sigma_{u}$ orbital, which are expressed by a basis set of Slater-type orbitals. 


\section{An analysis in terms of electronic forces}

The total force acting on a nucleus $A$ in a homonuclear diatomic for any internuclear separation $R$ is given by (Bader and Chandra 1968; Chandra and Sunder 1971; Sunder and Chandra 1974; Chandra and Sebastian 1976 a, b)

$$
F_{A}(R)=\frac{Z}{R^{2}}\left(Z-f_{A}^{\rho}\right)
$$

where

$$
f_{A}^{\rho}=R^{2} \int \rho(r) \frac{\cos \theta_{A i}}{r_{A i^{2}}} d \tau
$$

is called the charge equivalent of the net electronic force on nucleus $A$ in the direction of the bond, $Z$ is the nuclear charge and $\rho(r)$, the molecular electron density at a point $r$ evaluated for the internuclear separation $R$. In order to understand the detailed course of events during the atomic interaction it is more useful to consider $f_{A}^{\rho}$ than the total force $F_{A}(R)$. In figure 1 is shown the variation of $f_{A}^{\rho}$

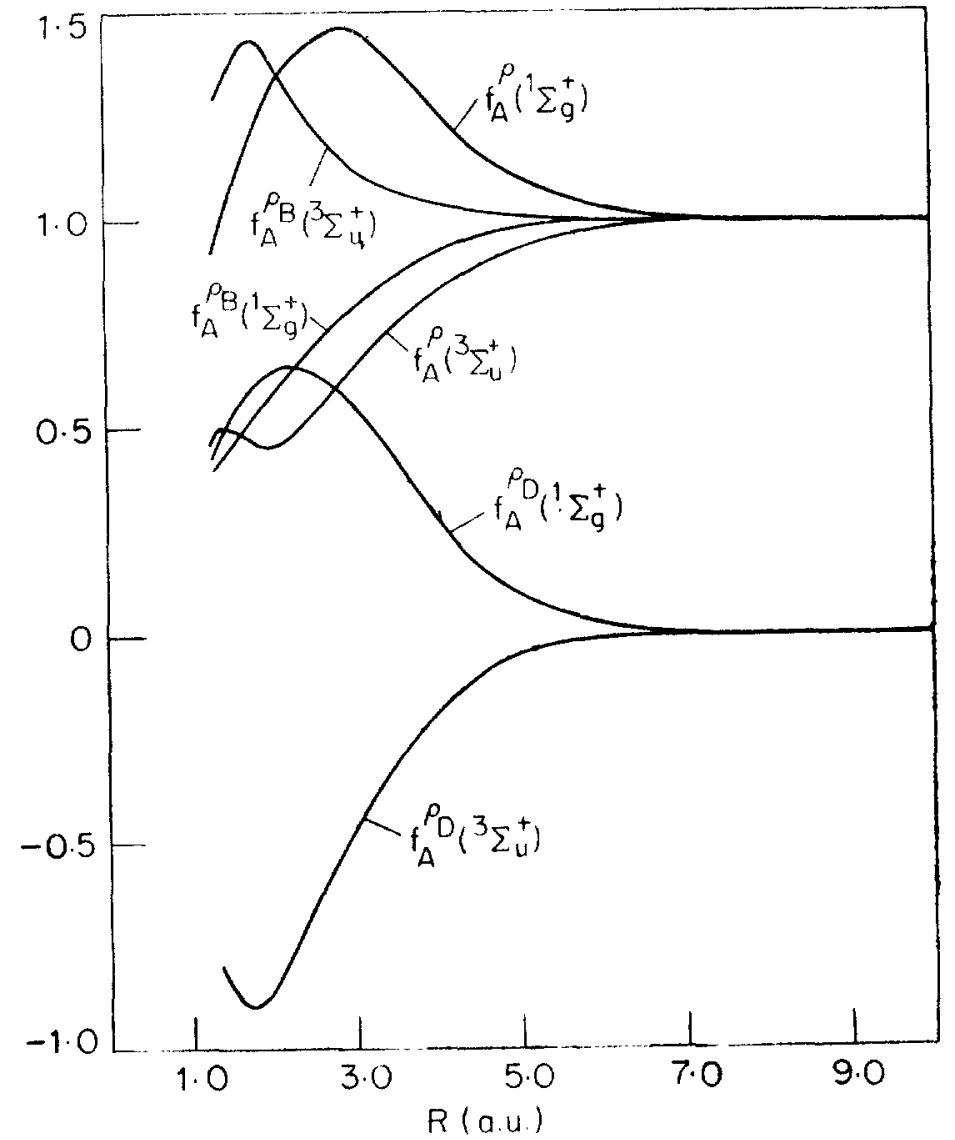

Figure 1. Variation with $R$ of $f_{A}^{\rho}\left({ }^{1} \Sigma_{q}^{+}\right), f_{A}^{\rho}\left({ }^{3} \Sigma_{u}^{+}\right), f_{A}^{\rho_{B}}\left({ }^{1} \Sigma_{v}^{+}\right), f_{A^{B}}\left({ }^{(9} \Sigma_{u}^{+}\right) f_{A}^{\rho_{D}}\left({ }^{1} \Sigma_{q}^{+}\right)$ and $f_{A}^{\rho} D\left({ }^{3} \Sigma_{u}^{+}\right)$. All the quantities are in atomic units. 
$\left({ }^{1} \Sigma_{\sigma}^{+}\right)$and $f_{A}^{\rho}\left({ }^{3} \Sigma_{u}^{+}\right)$with $R$. The limiting value of $f_{A}^{\rho}$ for both the singlet and triplet states of $\mathrm{H}_{2}$ is unity (Bader and Chandra 1968; Chandra and Sunder 1971; Sunder and Chandra 1974; Chandra and Sebastian $1976 \mathrm{a}, \mathrm{b}$ ). Figure 1 shows that at large $R$ the singlet and triplet states of $\mathrm{H}_{2}$ behave identically and such situation persists for $R \approx 7 a_{0}$. For $R<7 a_{0}$, the density in the ${ }^{1} \Sigma_{g}^{+}$state becomes more and more binding corresponding to a net force of attraction and the formation of a stable molecule, while in the ${ }^{3} \Sigma_{u}^{+}$state the charge distribution becomes antibinding corresponding to a net force of repulsion. From the comparison of the potential energy curves of the singlet and triplet states of $\mathrm{H}_{2}$, Bowman et al (1970) concluded that the near identity of these two states of $\mathrm{H}_{2}$ persists for $R$ as small as $5 a_{0}$. Our analysis has however revealed that these two states of $\mathrm{H}_{2}$ behave differently for $R>5 a_{0}$. This difference becomes more striking when we partition the electronic forces into three components as

$$
f_{A}=f_{A}^{\rho_{A}}+f_{A}^{\rho_{B}}+f_{A}^{\rho_{D}}
$$

and analyse each term separately. The three terms of equation (3) are respectively called the atomic polarisation, screening and the overlap (or delocalisation) terms and their physical significance has been discussed in earlier publications (Bader and Chandra 1968; Chandra and Sunder 1971; Sunder and Chandra 1974; Chandra and Sebastian 1976a, b). In figures 1 and 2 are shown the variation of these three

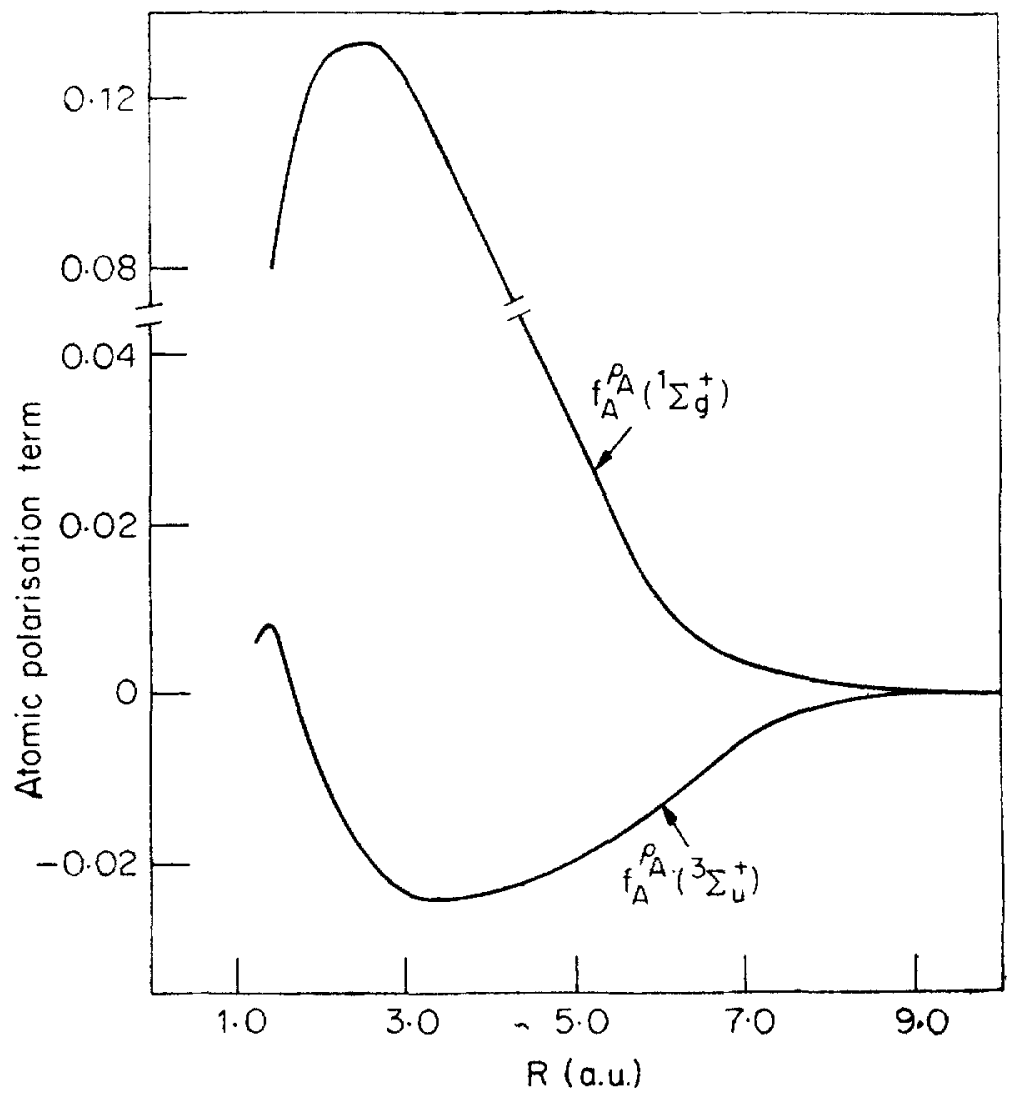

Figure 2. Variation with $R$ of $f_{A}^{\rho_{A}}\left({ }^{(} \Sigma_{0}^{+}\right)$and $f_{A}^{\rho_{A}}\left({ }^{3} \Sigma_{w}^{+}\right)$ 
terms with $R$. It should be noted that all these three terms, i.e., $f_{A}^{\rho_{A}}, f_{A}^{\rho_{B}}$ and $f_{A}^{\rho_{D}}$ should converge to zero in the limit of the united atom. The variation of these terms with $R$ in figures 1 and 2 shows this trend at a very small value of $R$.

The variation of $f_{A}^{\rho_{A}}$ with $R$ for both the ${ }^{3} \Sigma_{u}^{+}$and ${ }^{1} \Sigma_{g}^{+}$states (figure 2) shows that even for $R \geqslant 5 a_{0}$ when the overlap of two atomic orbitals is negligibly small, the electron cloud around each nucleus is polarised behind the nucleis in the ${ }^{3} \sum_{z}^{+}$ state. On the other hand, in the ${ }^{1} \Sigma_{j}^{+}$state each atomic density is polarised towards the internuclear region resulting in an attractive force on each nucleus. But the variation of $f_{A_{A}}^{\rho}\left({ }^{3} \Sigma_{u}^{+}\right)$with $R$ for $R<2 a_{0}$ is somewhat surprising. Instead of going over directly to zero at $R=0$, the value of $f_{A}^{\rho_{A}}\left({ }^{3} \Sigma_{u}^{+}\right)$rises to a positive value and then deareases to zero. This can be understood when we discuss the behaviour of the component $\left(\sigma_{0}\right.$ and $\sigma_{u}$ ) orbitals for various $R$.

When $R$ is decreased below $5 a_{0}$, the variation of $f_{A}^{\rho_{B}}$ with $R$ (figure 1 ) shows that in the singlet state each nucleus readily penetrates in to the charge cloud of the other ato $m$ while in the triplet state there is no evidence of such penetration. The increase of the screening term for the ${ }^{3} \Sigma_{t}^{+}$state over the limiting value can be caused either by the contraction of the atomic orbitals or by a transfer of densities from the internuclear region or by both. This increase of the screening term beyond the limiting value implies an attraction between two atoms in the ${ }^{3} \Sigma_{u}^{+}$state but this increase is small and more than offset by the highly repulsive overlap term.

The variation of the overlap term, i.e. $f_{A}^{\rho_{D}}$ with $R$ (fig ure 1 ) shows that as the two approaching atomic orbitals begin to overlap $f_{A}^{\rho_{D}}$ increases in the ${ }^{1} \Sigma_{g}^{+}$state implying transfer of charge in the internuclear region until it reaches a maximum. On further decrease of $R$, the charge density is transferred behind the nuclei causing a decrease in the value of $f_{A}^{\rho_{\mathrm{D}}}\left({ }^{1} \Sigma_{y}^{+}\right)$for $R<2 a_{0}$. On the other hand, in the ${ }^{3} \Sigma_{t}^{+}$ state, the variation of $f_{A} \rho_{D}$ with $R$ implies a transfer of charge from the internuclear region to the region behind the nuclei and this charge density exerts a force which pulls the nuclei away from one another. At $R=2 a_{0}$ the force of repulsion exerted by the overlap density in the ${ }^{3} \Sigma_{*}^{+}$state corresponds to an increase of the nuclear charge by about +0.85 units. This sizable effect arises from the fact that two electrons with their parallel spins cannot occupy the same region of space simultaneously. On further decrease of $R$, the value of $f_{A}^{\rho_{D}}\left({ }^{3} \Sigma_{w}^{+}\right)$increases. This may be due to the expansion of an orbital in which case the magnitude of the force on the nucleus should decrease or some charge density migrates to the internuclear region from the antibinding regions.

\section{Behaviour of $\sigma_{\mathfrak{v}}$ and $\sigma_{\mathfrak{u}}$ orbitals}

According to the simple concept of the molecular orbital theory for homopolar diatomics the $\sigma_{o}$ and $\sigma_{\mathrm{s}}$ orbitals are respectively binding and antibinding. Since the antibinding character of the $\sigma_{u}$ orbital density dominates, the ${ }^{1} \Sigma_{0}^{+}$state of $\mathrm{H}_{2}$ with the predominant configuration of ||$\sigma_{0}^{2} \mid$ is stable and the ${ }^{3} \Sigma_{u}^{+}$state with the configuration of $\left|! \sigma_{g}^{1}{ }^{\prime} \sigma_{u}^{1}\right|$ is unstable. This simple picture is however, only qualitatively valid when the force concept is used.

The total electronic force operative in the singlet and triplet states of $\mathrm{H}_{2}$ may be broken down into the orbital components. Thus

$$
f_{A}^{\rho}=f_{A}^{\sigma_{D}}+f_{A}^{\sigma_{u}}
$$


where $f_{A}^{i}$ is the charge equivalent of the partial force exerted on the nucleus $A$ by the density in the $i$ th molecular orbital. For the separated atoms the density distribution corresponding to cach non-degenerate molecular orbital $\varphi_{i}$ may be written as

$$
\varphi_{i}^{*} \varphi_{i}=\frac{1}{2}\left(\varphi_{i A}^{\prime \prime}+\varphi_{i B}^{2}\right),
$$

where $\varphi_{i A}$ and $\varphi_{i B}$ are the centrosymmetric atomic orbitals on atoms $A$ and $B$ with which $\varphi_{i}$ correlates for the separated atoms. At the limit of large $R, f_{A}^{i}$ becomes (Bader and Chandra 1968; Chandra and Sunder 1971; Sunder and Chandra 1974; Chandra and Sebastian 1976a,b)

$$
f_{A}^{i}(R=\infty)=n_{2} / 2,
$$

where $n_{i}$ is the occupation number of the $i$ th MO. In both the singlet and triplet states of $\mathrm{H}_{2}$ at $R=\infty$ the occupation number of the $\sigma_{q}$ and $\sigma_{u}$ orbitals is unity and therefore the limiting value of each $f_{A}^{i}$ is clearly 0.5 . In figure 3 are shown the

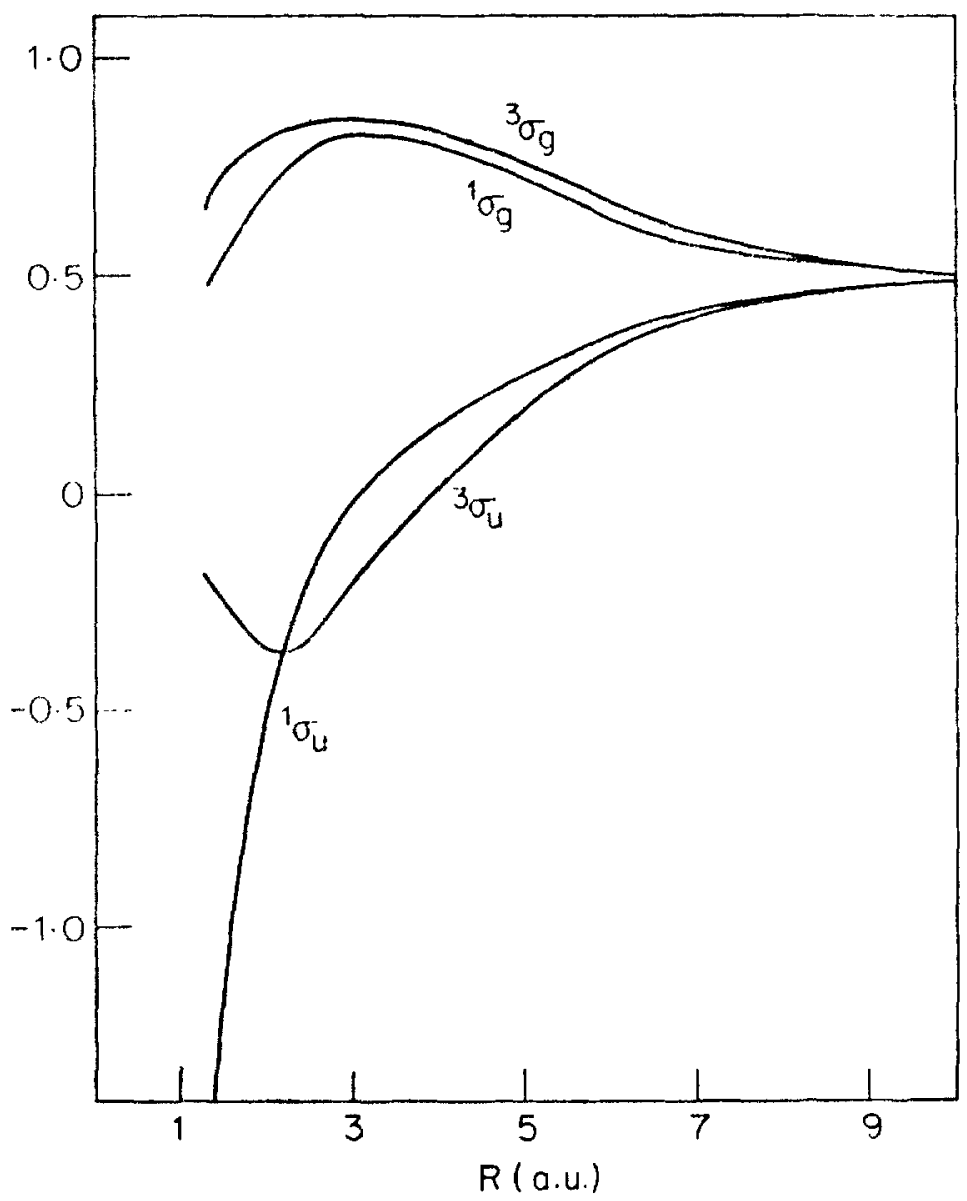

Figure 3. Variation with $R$ of $f_{A}^{\sigma} a$ and $f_{A}^{\sigma_{u}}$ per electron for the singlet and triplet states. 
variations of $f_{A}^{\sigma_{o}}$ and $f_{A}^{\sigma_{u}}$ per electron with $R$ for the singlet and triplet states. The results clearly show that ${ }^{1} \sigma_{g}$ and ${ }^{3} \sigma_{g}$ orbital densities are binding while ${ }^{1} \sigma_{*}$ and ${ }^{3} \sigma_{w}$ orbital densities are antibinding for $R<\infty$. The results further show that the properties of ${ }^{1} \sigma_{g}$ and ${ }^{3} \sigma_{g}$ orbital densities are similar for $R \geqslant 7 a_{0}$. For $R<7 a_{0}$, the ${ }^{3} \sigma_{v}$ orbital becomes more hinding than the ${ }^{1} \sigma_{v}$ orbital and this is possible if the ${ }^{3} \sigma_{o}$ orbital contracts relative to the ${ }^{1} \sigma_{g}$ orbital. This contraction of the ${ }^{3} \sigma_{g}$ orbital is more marked for $R<2 a_{0}$. However, both the ${ }^{1} \sigma_{0}$ and ${ }^{3} \sigma_{0}$ ortitals converge to $1 s$-orbital of helium at $R=0$.

On the other hand, although ${ }^{1} \sigma_{n}$ and ${ }^{3} \sigma_{u}$ orbitals behave similarly at large $R$, the difference between these two orbitals become increasingly marked at lower $R$ where the ${ }^{3} \sigma_{u}$ orbital becomes more antibinding than the ${ }^{1} \sigma_{u}$ orbital. For $R<$ $2 a_{0}$ the antibinding ability of the ${ }^{3} \sigma_{u}$ orbital density decreases while that of the ${ }^{1} \sigma_{u}$ density continues to increase. Since both the ${ }^{1} \sigma_{u}$ and ${ }^{3} \sigma_{u}$ orbitals go over to the $2 p$-orbital of helium at $R=0$, it suggests that the ${ }^{3} \sigma_{u}$ orbital expands relative to the ${ }^{1} \sigma_{u}$ density for $R<2 a_{0}$. Such expansion of the ${ }^{3} \sigma_{u}$ orbital relative to the ${ }^{1} \sigma_{u}$ orbital at $R=1 \cdot 5 a_{0}$ has been strikingly shown in the orbital density diagrams of Bowman et al (1970). It is however to be noted from figure 3 that there is no indication of $f_{A}^{1 \sigma_{u}}$ converging to zero in the limit of the united atom.

If we measure the binding and antibinding abilities of the $\sigma_{o}$ and $\sigma_{u}$ orbitals by the magnitude of $\left(0^{\cdot}:-\left|f_{A}^{i}\right|\right)$ then we see from figure 3 that for $R<7 a_{0}$ the antibinding character of the $\sigma_{2 t}$ orbitals dominates the binding abilities of the $\sigma_{p}$ orbitals. Since in the ${ }^{3} \Sigma^{+}$state the occupation number of the ${ }^{3} \sigma_{u}$ and ${ }^{3} \sigma_{v}$ orbitals is always unity at all $R$ while in the ${ }^{1} \sum_{j}^{+}$state the ocaupation of the ${ }^{1} \sigma_{u}$ orbital decreases at lower $R$ and has a value of approximately 0.02 (Bowman et al 1970) at $R=1 \cdot 4 a_{0}$, the ${ }^{1} \Sigma_{v}^{+}$state of $\mathrm{H}_{2}$ becomes stable at $R=1 \cdot 4 a_{0}$ and the ${ }^{3} \Sigma_{*}^{+}$state remains unstable at all values of $R$. Therefore, the instability of the ${ }^{3} \Sigma_{u}^{+}$state at small $R$ arises from the persistent tendency of its eleatrons to correlate while in the ${ }^{1} \Sigma_{0}^{+}$state such tendency of electrons is decreased so that two electrons can come close together on the average and exert a strong binding force on each nucleus.

One can now also understand why the atomic term in the triplet state increases to a positive value for $R<2 a_{0}$ and then decreases to zero as $R$ tends to zero. Normally, the $\sigma_{u}$ charge density exerts a negative atomic force which cancels the positive atomic term contributed by the $\sigma_{g}$ density. On account of the expansion of the $\sigma_{u}$ orbital the magnitude of its atomic term decreases while the positive atomic term of the $\sigma_{v}$ density increases due to its contraction.

\section{Changes in the charge distribution during the $\mathbf{H}+\mathbf{H}$ interactions}

The changing nature of the density distributions as the two $\mathrm{H}$-atoms approach one another resulting in the formation of the stable $\mathrm{H}_{2}$ molecule has already been illustrated in the $\triangle \rho$-contour maps (Bader and Chandra 19.8) employing the extended Hartree-Fock wave functions of Das and Wahl (1966). Similar $\triangle \rho$-diagrams are obtained for the ground-state of $\mathrm{H}_{2}$ from the wave functions of Bowman et al (1970) and are therefore not reported here. However, it will be of great interest to examine how the charge distribution changes in the triplet state of $\mathrm{H}_{2}$ as a function of $R$. Figure 4 presents $\Lambda \rho$-Gontour maps for the ${ }^{3} \Sigma_{n}^{+}$state of $\mathrm{H}_{2}$ at a number of internuclear distances calculated from the wave functions of Bowman et al (1970). 
Interaction in $\mathrm{H}_{2}$ singlet and triplet
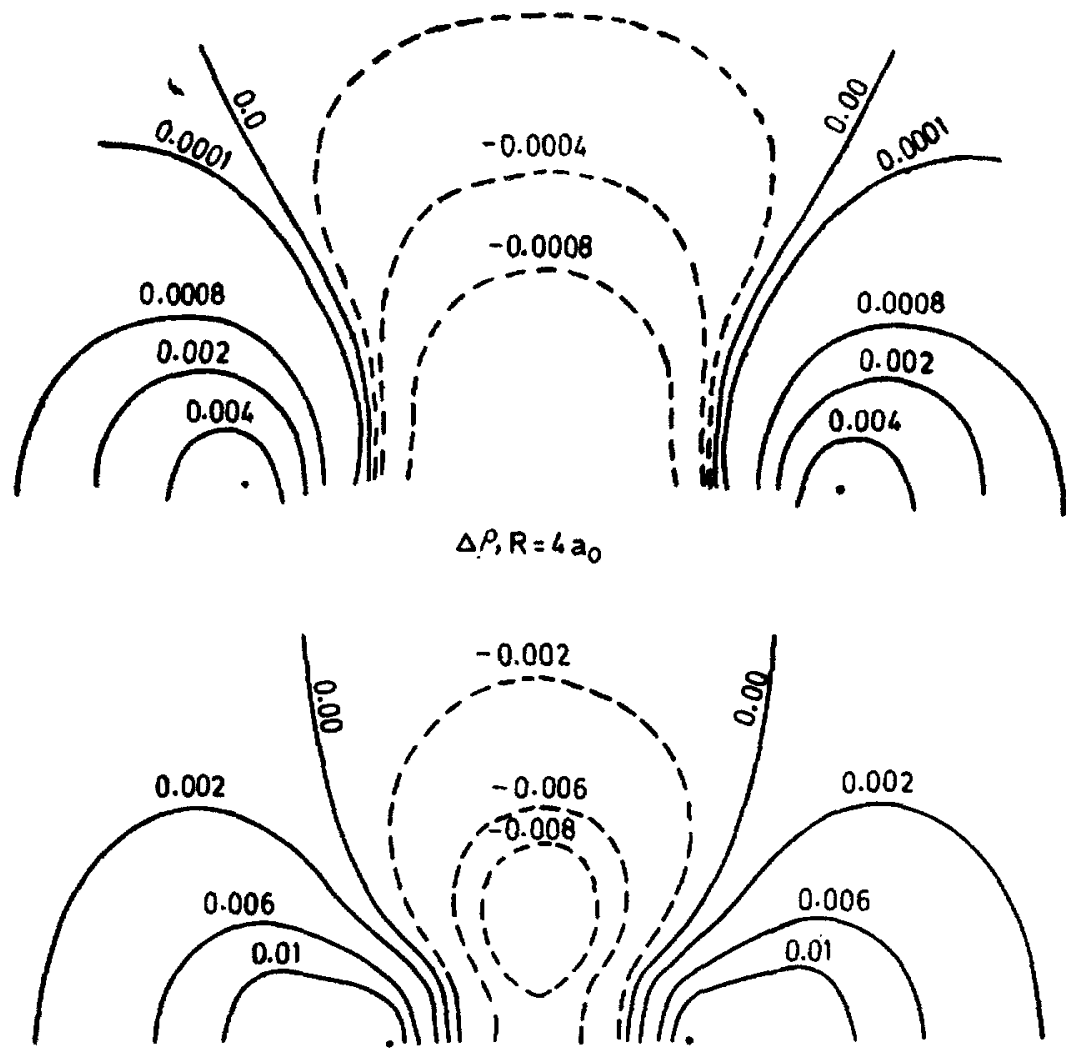

$\Delta P, R=2 a_{0}$

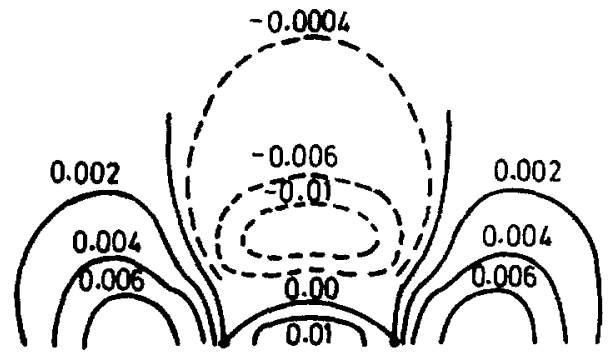

$\Delta P \cdot R=1.4 \mathrm{a}_{0}$

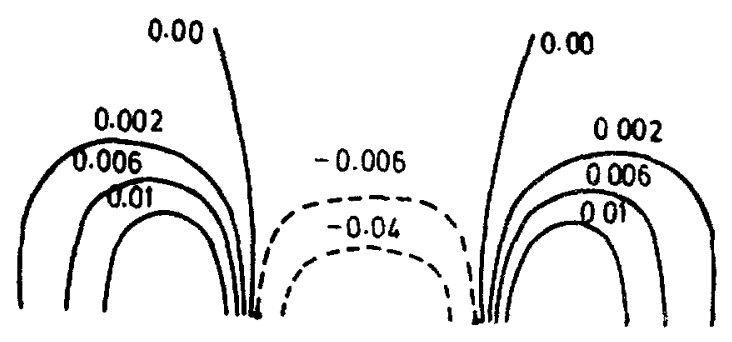

$$
\Delta P_{e}, R=1.4 \mathrm{a}_{0}
$$

Figure 4. The $\Delta \rho$-contour maps for the ${ }^{3} \sum_{1}^{+}$state of $\mathrm{H}_{2}$ at the indicated values of $R$ and the $\triangle \rho_{0}$-map for $R=1 \cdot 4 a_{0}$. 
These diagrams are the approximate opposites of the $\Delta \rho$-maps for the singlet state of $\mathrm{H}_{2}$. The densities in the ${ }^{3} \Sigma_{u}^{+}$state increase in the regions behind the nuclei as well as in the vicinity around each nucleus at the expense of densities in the internuclear region as $R$ is decreased. But, at $R=1 \cdot 4 a_{n}$, figure 4 shows that there is an indication of slight increase in density in the internuclear region. This could arise from the simultaneous expansion of the ${ }^{3} \sigma_{u}$ orbital and the contraction of the ${ }^{3} \sigma_{v}$ orbital both having the occupation number of unity each in the ${ }^{3} \Sigma_{u}^{+}$state. In fact, the small positive atomic term at $R=1 \cdot 4 a_{0}$ in figure 2 is consistent with the slight increase of density in the internuclear region. But this increase is very small and does not balance the nuclear repulsion, hence the ${ }^{3} \Sigma_{u}^{+}$state is unstable. Figure 4 also shows the plot of $\Delta \rho_{0}$ defined as

$$
\Delta \rho_{e}=\rho\left({ }^{3} \Sigma_{u}^{+}\right)-\rho\left({ }^{1} \Sigma_{g}^{+}\right) \text {, }
$$

for $R=1 \cdot 4 a_{0}$, where $\rho\left({ }^{3} \Sigma_{1}^{+}\right)$and $\rho\left({ }^{1} \Sigma_{g}^{+}\right)$are the molecular charge densities of the ${ }^{3} \Sigma_{u}^{+}$and ${ }^{1} \Sigma_{g}^{+}$states respectively for the same value of $R$. This shows that the charge density in the internuclear region is greater in the ${ }^{1} \Sigma_{y}^{+}$state than in the ${ }^{3} \Sigma_{u}^{+}$staté.

It may be important to mention here that a similar study on the triplet state of $\mathrm{HeH}^{+}$which is isoelectronic with the ${ }^{3} \Sigma_{u}^{+}$state of $\mathrm{H}_{2}$ as it dissociates into $\mathrm{He}^{+}$and $H$ has been made earlier (Chandra and Sebastian 1976a). It was shown that the triplet state of $\mathrm{HeH}^{+}$shows a weak binding due to large polarisation of the electron cloud of the $\mathrm{H}$ atom caused by the unscreened positive charge of the $\mathrm{He}^{+}$ion.

\section{Acknowledgements}

The authors thank Prof. J O Hirschfelder of the University of Wisconsin, Madison, USA, for sending us the detailed wave functions, the Department of Atomic Energy, Government of India for the financial support and the Computer Centre, Indian Institute of Science, for the computing facilities.

\section{References}

Bader R F W and Chandra A K 1968 Can. J. Chem. 46953

Bowman J D, Hirschfelder J O and Wahl A C 1970 J. Chem. Phys. 532743

Chandra A K and Sunder R 1971 Mol. Phys. 22369

Chandra A K and Sebastian K L 1976a Mol. Phys. 311489

Chandra A K and Sebastian K L 1976 b Chem. Phys. Lett, 41593

Das G and Wahl A C 1966 J. Chem. Phys, 4487

Sunder $R$ and Chandra A K 1974 Ittian J. Chem. 12145 